\title{
Ultrasonic Continuous-Wave Beam-Power Measurements; International Intercomparison
}

\author{
Carl E. Tschiegg, * Martin Greenspan,** and Donald G. Eitzen*
}

\author{
National Bureau of Standards, Washington, DC 20234
}

October 26, 1982

\begin{abstract}
Some quartz transducers designed and fabricated at the National Bureau of Standards as transmitters of ultrasonic power appear to be sufficiently stable and linear to serve as standards. Therefore, an international intercomparison of measurements of the continuous-wave (cw) power emitted by these standards was arranged. Each of the seven participating laboratories performed such measurements using one or more methods representing its practice and reported the results to the National Bureau of Standards which served as the pilot laboratory. We present the results mostly in the form of tables. Some remarks on stability are appended.
\end{abstract}

Key words: intercomparison of standards; ultrasonic power standards; ultrasonic transducers.

\section{Introduction}

In 1974, Thomas L. Zapf, [1] $]^{1}$ of the then Electromagnetics Division of the National Bureau of Standards in Boulder, CO, U.S.A., described a method for the measurement of the radiation conductance of an ultrasonic transducer by means of high-accuracy impedance measurements made with a twin-T null circuit. Also described were some quartz transducers designed and constructed so that they could be expected to be stable over long intervals of time. By the spring of 1975 , Helmut M. Altschuler of the Electromagnetics Division of NBS Boulder was actively arranging the international comparison of ultrasonic beam-power measurements utilizing the new standards; the technical direction of the project was to be Zapf's responsibility. In the fall of 1976, when many of the arrangements with the participating laboratories had been completed, the responsibility for the project was transferred to Donald G. Eitzen, chief of the then Ultrasonic Standards Program Team in Washington, as a result of a management decision to consolidate some of the work in ultrasonics at NBS. Also transferred, besides some equipment, were 14 quartz transducers, having operating frequencies of

\footnotetext{
*Mechanical Production Metrology Division, Center for Manufacturing Engineering, National Engineering Laboratory.

***Present address: I2 Granville Dr., Silver Spring, MD 20901.

'Figures in brackets denote literature references at the end of thits paper.
}

$2,3,5,10$, and $15 \mathrm{MHz}$. These were calibrated by the modulated radiation-pressure method [2] over the ranges of ultrasonic power output from 1 to $8 \mathrm{~mW}$ up to 1 to 528 $\mathrm{mW}$, and the transducers were found to be linear (i.e., power output proportional to square of voltage), so that each transducer could be characterized by a single radiation conductance $\left(G_{r}\right)$. Furthermore, this $G_{r}$ proved to be the same, within the estimated experimental errors, as that determined at very low power by the twin-T null method. Other transducers were also calibrated by a calorimetric method, using an instrument designed and built by Zapf et al. [3] at NBS Boulder and modified at NBS Washington ${ }^{2}$. Each transducer was calibrated (twice) at only one power level which varied, from case to case, over the range 50 to $750 \mathrm{~mW}$. Again, the values of $G_{r}$ agreed, within the estimated uncertainty, with those obtained by the other methods.

\section{Procedure}

Four ultrasonic transducers were selected for the intercomparison. They were intended to be operated at the fundamental series-resonance frequencies; these, together with the designations, follow:

\footnotetext{
'The modifications, which were rather extensive, were made by Franklin $R$. Breckenridge and Carl E. Tschiegg.
} 


\begin{tabular}{|c|c|c|}
\hline \multirow{3}{*}{ Standard No. } & \multicolumn{2}{|c|}{ Operating Frequency } \\
\hline & Nominal & Actual \\
\hline & $\mathbf{M H z}$ & $\mathbf{M H z}$ \\
\hline
\end{tabular}

$\begin{array}{lll}3-16 & 2 & 1.995 \\ 3-18 & 2 & 1.995 \\ 3-20 & 5 & 5.046 \\ 3-22 & 5 & 5.015\end{array}$

The active element of each was a half-wave resonant, air-backed, x-cut quartz disc, having a "wrap-around" outer electrode to provide some electrostatic shielding. The discs were cut, polished, and plated (gold over chromium) by a commercial supplier. The transducers are shown in figure 1 .

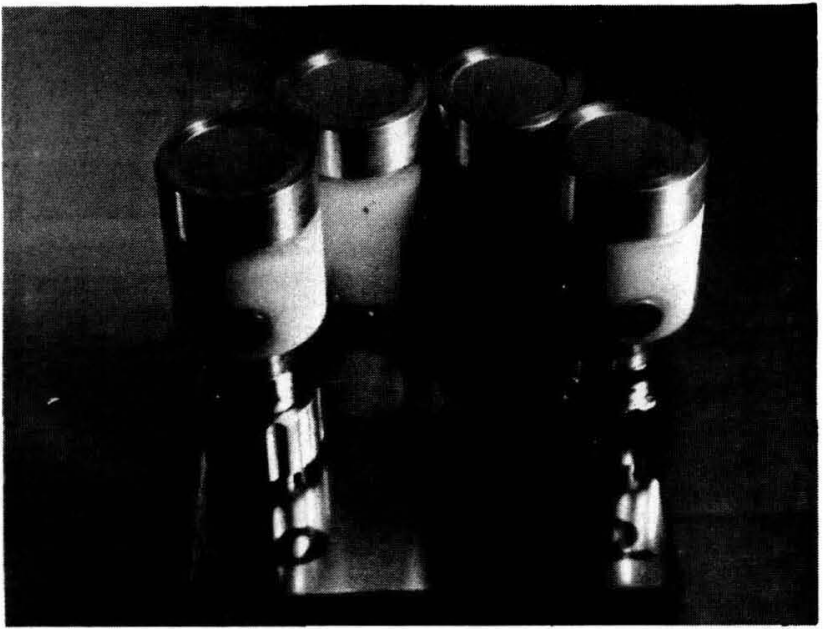

FigurE 1. The four standard transducers.

The transducers were shipped to the participating laboratories and the measurements made in the following order:

1. National Bureau of Standards Boulder, CO, U.S.A.

2. National Bureau of Standards Washington, DC, U.S.A.

3. Radiation Protection Bureau Ottawa, Ontario, Canada

4. National Research Council Ottawa, Ontario, Canada
5. NBS (Remeasurement)

6. Bureau of Radiological Health Rockville, MD, U.S.A.

7. NBS (Remeasurement)

8. Ultrasonics Institute Sydney, Australia

9. NBS (Remeasurement)

10. Physikalisch-Technische Bundesanstaldt Braunschweig, FRG

11. NBS (Remeasurement)

12. National Physical Laboratory Teddington, Middlesex, U.K.

\section{NBS (Remeasurement)}

The instructions to the participating laboratories are reproduced in the Appendix. Each laboratory used its own method or methods, a total of eight. The methods are listed together with their code designations in table 1.

TABLE 1. Methods of ultrasonic cw beam power measurement and codes.

\begin{tabular}{ll}
\hline Method & Code \\
\hline & \\
Radiation balance, direct & RD \\
Radiation balance, feedback & RF \\
Radiation balance, modulated & RM \\
Calorimeter & $\mathbf{C}$ \\
Optical (Raman-Nath) & $\mathbf{O}$ \\
Impedance, twin-T null & $\mathbf{T}$ \\
Impedance, Q-meter & $\mathbf{Q}$ \\
Reciprocity & $\mathbf{R}$ \\
\hline
\end{tabular}

The voltage levels and tolerances specified in the Appendix were selected to cover ultrasonic power levels roughly as follows:

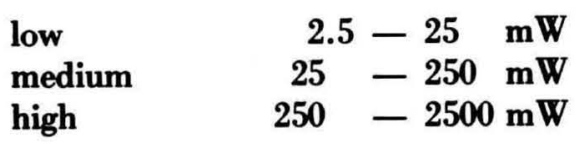




\section{Results}

The results are given in tables $2,3,4$, and 5 for transducers $3-16,18,20$, and 22 , respectively. In the column headed "Code" the arabic numeral following the method designation (as in e.g., RD-l) denotes one of the participating laboratories. In cases in which a laboratory used several methods, the associated numeral is different for each method. Each laboratory was furnished a key that enabled it to identify its own work, but not that of others. An exception is the pilot laboratory, NBS, for which RM-1 and T-1 are used.

In these tables, $U_{v}$ is the fractional uncertainty in the measurement of input voltage, and $U_{b}$ is that in the radiation conductanee, taking into account that in the volage. See the Appendix for details. Each investigator, acting in accordance with instructions, estimated these uncertainties by his own methods, so that the several laboratories have not reported on a common basis.

Inspection of tables $2,3,4$, and 5 reveals that by and large, there is rather remarkable agreement in the measurement of ultrasonic beam power at the power levels specified amang the several laboratories and methads (or coses )." In order to see this better, it is convenient to look at the departure of each result from some sort of grand average of all results. The question is how to weight the individual averages of the radiation conductance $\bar{G}_{r}$, in the calculation of the grand average, $\overline{\bar{G}}_{r^{*}}$. Our first thought was to use weights inversely proportional to the estimated errors, but we discarded this because, as has been pointed out, the reported errors are not comparable. We eventually decided to use purely statistical weights, that is, weights equivalent to the reciprocal variances. However, it was easier to use an approximation to the variance calculated from the range (max-min in tables 2, 3, 4, and 5) and the mean value of the ratio of the range to the standard deviation fsqnare root of the rariancel; this ratio depends on the number of data. Values of it are given in table 2.4.1 of Snedecor [4]. No deep significance is attached to the grand averages, $\overline{\bar{G}}_{\mathrm{r}}$; we present them as being perhaps good enough for the present purpose. Data for which the ranges were not given were not used in the calculation of the grand average, nor were those averages used which were calculated from fewer than three data. The deviations from the grand average were given in table 6 .

\footnotetext{
"Some of the methods used by the perticipating laboratories, e.g*, steady radiation pressure and calorimetric, are in principal capable of measuring average pulsed power. In some applications, auch as medical imaging, such average powers might be much lower than those used in these tests and the conclusion would not apply.
}

We can consolidate the material given in table 6 in the form of table $\bar{t}$, in which the number of cases where the absolute value of the deviation, $\left|\left(\bar{G}_{r}-\overline{\bar{G}}_{r}\right) / \bar{G}_{r}\right|$ is less than various amounts (in percent). A method of measurement by a particular laboratory forms a case, and the results are presented separately for the two nominal frequencies; the values for transducers 3-16 and $3-18$ are lumped in one column and those for $3-20$ and $3-22$ in the other. We see that at $2 \mathrm{MHz}$ the deviations are less than 1 percent in 11 percent of the cases and less than 3 percent in 33 percent of the cases. For $5 \mathrm{MHz}$ the peak is sharper; the values are 40 and 80 percent of the cases, respectively. At both frequencies, more than 90 pereent of the cases have $\bar{G}_{r}$ within 9 percent of $\overline{\vec{G}}_{r}$ and if one case (RD-3) were ignored, the figure would be 100 pereent.

We can only speculate as to why the data are better at 5 than at $2 \mathrm{MHz}$. Perhaps the increased absorption at 5 $\mathrm{MHz}$ eases the requirements on the anechoic materials, the performance of which is almost always less than one might hope.

It is worth noting that in each case except one (RD-3) the deviation laf the overall average for a case from the grand averagel is less than the experimenter's extimate of the error. This would mean little if in most of the cases the results were all too high or all too low. But in consideration of the diversity of the methods employed, this is not likely, and it would seem that most of the experimenters have made conservative estimates of their errors.

The results are, on the whole, gratifying. They lead us to believe that one can really measure with acceptable accuracy the total cw sound power output of transducers in the frequency range $2-5 \mathrm{MHz}$ and in the power range $2.5-2500 \mathrm{~mW}$. Unfortunately, the same conclusion cannot be extended into the fractional milliwatt range that is important to applications (such as medical diagnosis) characterized by high peak but low average power. The prospects for extending agreement to microwalt levels of average power are probahly good.

\section{Stability}

As shown in table 3 and its footnote $n$, one of the participants noticed a substantial change, between runs, of the value of $G_{r}$ for transducer 3-18. Extensive measurement $₫$ on this transdwoer were made upon its return to the pilot laboratory, but no significant changes from the pristine value were detected even after a three-day submersion of the operating face in water.

We have had a similar experience ourselves. A transducer of nominally identical construction as 3-18 
TABLE 2. Transfer standard No. 3-16 nominal frequency $2 \mathrm{MHz}$.

\begin{tabular}{|c|c|c|c|c|c|c|c|c|c|}
\hline \multirow[t]{3}{*}{ Code $^{a}$} & \multirow[t]{3}{*}{$\begin{array}{l}\text { Power } \\
\text { Range }\end{array}$} & \multirow[t]{3}{*}{$\begin{array}{r}\text { No. of } \\
\text { Data }\end{array}$} & \multicolumn{3}{|c|}{ Applied Voltage } & \multicolumn{4}{|c|}{ Radiation Conductance } \\
\hline & & & $\underline{\text { Min }}$ & $\underline{\operatorname{Max}}$ & $\mathrm{U}_{\mathrm{V}}$ & Min & $\underline{\text { Max }}$ & Avg. & $\mathbf{U}_{\mathbf{b}}$ \\
\hline & & & $\mathrm{V}$ & $\mathrm{V}$ & $\pm \%$ & $\mu \mathrm{S}$ & $\mu \mathrm{S}$ & $\mu S$ & $\pm \%$ \\
\hline \multirow[t]{4}{*}{ RD-1 } & low & 5 & 30 & 30 & 0.25 & 9 & 12 & 11 & 22 \\
\hline & med & 5 & 100 & 100 & 0.25 & 11.5 & 11.9 & 11.6 & 7 \\
\hline & high & 5 & 180 & 180 & 0.25 & 12.0 & 12.2 & 12.1 & 5 \\
\hline & overall & 15 & 30 & 180 & - & 9 & 12.2 & 11.54 & - \\
\hline \multirow[t]{4}{*}{ RD-2 } & low & 3 & 35 & 35 & 1.3 & 11.7 & 11.8 & 11.8 & 10 \\
\hline & med & 12 & 56 & 71 & 1.3 & 11.2 & 11.9 & 11.5 & 10 \\
\hline & high & 0 & - & - & - & - & - & - & - \\
\hline & overall & 15 & 35 & 71 & - & 11.2 & 11.9 & 11.58 & - \\
\hline \multirow[t]{4}{*}{ RD-3 } & low & 10 & 30 & 30 & 0.5 & 24.9 & 38.5 & 29.9 & $?^{\mathrm{c}}$ \\
\hline & med & 10 & 100 & 100 & 0.5 & 10.4 & 12.6 & 10.9 & $7^{\mathrm{c}}$ \\
\hline & high & 10 & 180 & 180 & 0.5 & 11.5 & 12.6 & 12.00 & $2.8^{\mathrm{c}}$ \\
\hline & overall & 30 & 30 & 180 & - & 10.4 & 38.5 & $17.6^{\mathrm{d}}$ & - \\
\hline \multirow[t]{4}{*}{ RD-4 } & low & 1 & $35^{e}$ & 35 & 2 & 9.3 & 9.3 & 9.3 & 19 \\
\hline & med & 2 & 53 & 71 & $2^{\mathrm{c}}$ & 10.8 & 11.7 & 11.2 & 19 \\
\hline & high & 0 & - & 一 & - & - & - & - & - \\
\hline & overall & 3 & 35 & 71 & - & 9.3 & 11.7 & 10.6 & - \\
\hline \multirow[t]{4}{*}{ RF-1 } & low & 6 & 26 & 26 & 0.2 & 10.6 & 11.2 & 10.9 & $6.1^{\mathrm{c}, f}$ \\
\hline & med & 6 & 81 & 82 & 0.2 & 10.9 & 11.2 & 11.0 & $5.6^{\mathrm{c}, \mathrm{f}}$ \\
\hline & high & 7 & 254 & 259 & 1.0 & 11.1 & 11.8 & 11.4 & $7.6^{c, f}$ \\
\hline & overall & 19 & 26 & 259 & - & 10.6 & 11.8 & 11.13 & 一 \\
\hline \multirow[t]{4}{*}{ RF-3 } & low & 3 & 35 & 35 & 1.3 & 11.7 & 11.8 & 11.7 & 10 \\
\hline & med & 6 & 56 & 70 & 1.3 & 11.9 & 12.0 & 11.9 & 10 \\
\hline & high & 0 & - & - & - & - & - & - & - \\
\hline & overall & 9 & 35 & 70 & - & 11.7 & 12.0 & 11.86 & - \\
\hline \multirow[t]{4}{*}{ RF-4 } & low & $9 / 20^{8}$ & 26 & 36 & 0.3 & 10.3 & 11.2 & 10.9 & 3.8 \\
\hline & med & $9 / 20$ & 66 & 91 & 0.4 & 10.4 & 11.3 & 10.9 & 3.9 \\
\hline & high & $9 / 20$ & 146 & 187 & 0.5 & 10.5 & 11.2 & 10.9 & 4.2 \\
\hline & overall & $27 / 60$ & 26 & 187 & - & 10.3 & 11.3 & 10.87 & - \\
\hline \multirow[t]{4}{*}{ RM-1 } & low & 34 & 16 & 46 & 0.2 & 11.2 & 11.5 & 11.4 & 2.3 \\
\hline & med & 15 & 66 & 135 & 0.2 & 11.3 & 11.3 & 11.3 & 2.3 \\
\hline & high & 17 & 150 & 216 & 0.2 & 11.3 & 11.3 & 11.3 & 2.3 \\
\hline & overall & 66 & 16 & 216 & - & 11.2 & 11.5 & 11.34 & - \\
\hline C.-3 ${ }^{h}$ & i & $\mathbf{i}$ & $\mathbf{i}$ & i & & i & $\mathbf{i}$ & 11.1 & 7 \\
\hline \multirow[t]{4}{*}{ C-5 } & low & 3 & 35 & 36 & 1.3 & 11.3 & 12.3 & 11.8 & 10 \\
\hline & med & 3 & 59 & 61 & 1.3 & 11.8 & 12.4 & 12.1 & 10 \\
\hline & high & 0 & - & - & - & - & - & - & - \\
\hline & overall & 6 & 35 & 61 & - & 11.3 & 12.4 & 11.94 & - \\
\hline \multirow[t]{4}{*}{0,1} & low & 0 & - & - & 一 & - & - & - & - \\
\hline & med & 9 & 70 & 141 & 1.3 & 10.6 & 12.1 & 11.3 & 10 \\
\hline & high & 5 & 176 & 211 & 1.3 & 10.9 & 11.7 & 11.3 & 10 \\
\hline & overall & 14 & 70 & 211 & - & 10.6 & 12.1 & 11.29 & - \\
\hline$Q-4$ & j & 10 & $\mathbf{j}$ & $\mathbf{j}$ & $\mathbf{i}$ & i & $\mathbf{i}$ & 10.56 & $\mathbf{i}$ \\
\hline \multirow[t]{5}{*}{$\mathrm{R}-2^{k}$} & low & 6 & 30 & 38 & $\mathbf{i}$ & 10.9 & 11.5 & 11.1 & 17 \\
\hline & med & 0 & - & - & - & - & - & - & \\
\hline & high & 0 & - & - & 一 & - & - & 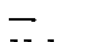 & \\
\hline & overall & 6 & 30 & 38 & & 10.9 & 11.5 & 11.1 & \\
\hline & & & \multicolumn{3}{|c|}{ weighted mean ${ }^{P}$} & & & 11.44 & \\
\hline
\end{tabular}


TABLE 3. Transfer standard No. 3-18 nominal frequency $2 \mathrm{MHz}^{*}$.

\begin{tabular}{|c|c|c|c|c|c|c|c|c|c|}
\hline \multirow[t]{3}{*}{ Code $^{a}$} & \multirow[t]{3}{*}{$\begin{array}{l}\text { Power } \\
\text { Range }\end{array}$} & \multirow[t]{3}{*}{$\begin{array}{r}\text { No. of } \\
\text { Data }\end{array}$} & \multicolumn{3}{|c|}{ Applied Voltage } & \multicolumn{4}{|c|}{ Radiation Conductance } \\
\hline & & & $\underline{\text { Min }}$ & $\underline{\text { Max }}$ & $\mathrm{U}_{\mathrm{V}}$ & $\underline{\text { Min }}$ & $\underline{\operatorname{Max}}$ & Avg. & $\mathbf{U}_{\mathbf{b}}$ \\
\hline & & & $\bar{V}$ & $\overline{\mathrm{V}}$ & $\overline{ \pm \%}$ & $\overline{\mu S}$ & $\overline{\mu S}$ & $\mu \mathrm{S}$ & $\pm \%$ \\
\hline \multirow[t]{4}{*}{ RD-1 } & low & 5 & 30 & 30 & 0.25 & 8 & 10 & 9 & 22 \\
\hline & med & 5 & 100 & 100 & 0.25 & 11.1 & 11.2 & 11.2 & 7 \\
\hline & high & 5 & 180 & 180 & 0.25 & 11.7 & 11.9 & 11.8 & 5 \\
\hline & overall & 15 & 30 & 180 & - & 8 & 11.9 & 10.66 & - \\
\hline \multirow[t]{4}{*}{ RD-2 } & low & 3 & 35 & 35 & 1.3 & 11.8 & 11.9 & 11.9 & 10 \\
\hline & med & 12 & 56 & 71 & 1.3 & 11.2 & 11.8 & 11.5 & 10 \\
\hline & high & 0 & - & - & - & - & - & - & - \\
\hline & overall & 15 & 35 & 71 & - & 11.2 & 11.9 & 11.57 & - \\
\hline \multirow[t]{4}{*}{ RD-3 } & low & 10 & 30 & 30 & 0.5 & 16.3 & 43.0 & 31.4 & $35^{\mathrm{c}}$ \\
\hline & med & 10 & 100 & 101 & 0.5 & 12.6 & 14.0 & 13.2 & $6.7^{\mathrm{c}}$ \\
\hline & high & 10 & 180 & 180 & 0.5 & 11.2 & 11.5 & 11.3 & 3.0 \\
\hline & overall & 30 & 30 & 180 & - & 11.2 & 43.0 & $18.64^{\mathrm{m}}$ & - \\
\hline \multirow[t]{4}{*}{ RD-4 } & low & 1 & $35^{e}$ & 35 & 2.0 & 10.2 & 10.2 & 10.2 & 18 \\
\hline & med & 2 & 53 & 71 & $1.9^{c}$ & 10.5 & 11.4 & 11.0 & $19^{c}$ \\
\hline & high & 0 & - & - & - & - & - & - & - \\
\hline & overall & 3 & 35 & 71 & - & 10.2 & 11.4 & 10.7 & - \\
\hline \multirow[t]{4}{*}{ RF-1 } & low & 7 & 25 & 26 & 0.2 & 10.0 & 10.8 & 10.4 & $5.5^{\mathrm{c}, \mathrm{f}}$ \\
\hline & med & 7 & 81 & 82 & 0.2 & 10.0 & 10.7 & 10.3 & $5.6^{\mathrm{c}, \mathrm{f}}$ \\
\hline & high & 7 & 254 & 256 & 1 & 10.5 & 11.1 & 10.7 & $7.6^{\mathrm{c}, \mathrm{I}}$ \\
\hline & overall & 21 & 25 & 256 & - & 10.0 & 11.1 & 10.45 & - \\
\hline \multirow[t]{4}{*}{ RF-3 } & low & 3 & 35 & 35 & 1.3 & 11.0 & 11.3 & 11.2 & 10 \\
\hline & med & 6 & 56 & 70 & 1.3 & 11.4 & 11.5 & 11.4 & 10 \\
\hline & high & 0 & - & - & - & - & - & - & - \\
\hline & overall & 9 & 35 & 70 & - & 11.0 & 11.5 & 11.35 & - \\
\hline \multirow[t]{4}{*}{ RF4. $1^{\mathrm{n}}$} & low & $7 / 26^{g}$ & 21 & 36 & 0.3 & 11.2 & 12.5 & 11.7 & 3.8 \\
\hline & med & $7 / 26$ & 72 & 97 & 0.4 & 11.1 & 11.7 & 11.4 & 3.9 \\
\hline & high & $6 / 22$ & 160 & 296 & 0.5 & 11.8 & 12.2 & 11.9 & 4.2 \\
\hline & overall & $20 / 74$ & 21 & 296 & - & 11.1 & 12.5 & 11.65 & - \\
\hline \multirow[t]{4}{*}{$\mathrm{RF} 4.2^{\mathrm{n}}$} & low & $8 / 20^{8}$ & 22 & 32 & 0.3 & 10.3 & 11.0 & 10.7 & 3.8 \\
\hline & med & $8 / 20$ & 70 & 97 & 0.4 & 10.3 & 10.9 & 10.6 & 3.9 \\
\hline & high & $8 / 20$ & 140 & 191 & 0.5 & 10.4 & 10.9 & 10.7 & 4.2 \\
\hline & overall & $24 / 60$ & 22 & 191 & - & 10.3 & 11.0 & 10.65 & - \\
\hline \multirow[t]{4}{*}{ RM-1 } & low & 32 & 16 & 36 & 0.2 & 11.0 & 11.3 & 11.2 & 2.3 \\
\hline & med & 13 & 46 & 150 & 0.2 & 11.0 & 11.2 & 11.1 & 2.3 \\
\hline & high & 19 & 151 & 232 & 0.2 & 11.0 & 11.4 & 11.2 & 2.3 \\
\hline & overall & 64 & 16 & 232 & - & 11.0 & 11.4 & 11.16 & - \\
\hline \multirow[t]{4}{*}{ C-5 } & low & 3 & 35 & 37 & 1.3 & 11.3 & 11.9 & 11.5 & 10 \\
\hline & med & 3 & 56 & 58 & 1.3 & 10.9 & 12.2 & 11.5 & 10 \\
\hline & high & 0 & - & - & - & - & - & - & - \\
\hline & overall & 6 & 35 & 58 & - & 10.9 & 12.2 & 11.50 & - \\
\hline \multirow[t]{4}{*}{$0-1$} & low & 0 & - & - & - & - & - & - & - \\
\hline & med & 9 & 70 & 141 & 1.3 & 10.8 & 11.9 & 11.4 & 10 \\
\hline & high & 6 & 176 & 211 & 1.3 & 10.8 & 11.5 & 11.3 & 10 \\
\hline & overall & 15 & 70 & 211 & - & 10.8 & 11.9 & 11.33 & - \\
\hline Q-4 & j & 10 & j & $\mathbf{j}$ & $\mathbf{i}$ & i & $\mathbf{i}$ & 10.24 & i \\
\hline
\end{tabular}


(Continuation) Table 3. Transfer standard No. 3-18 nominal frequency $2 \mathrm{MHz}$.

\begin{tabular}{|c|c|c|c|c|c|c|c|c|c|}
\hline \multirow[t]{2}{*}{$\operatorname{Code}^{a}$} & \multirow[t]{2}{*}{$\begin{array}{l}\text { Power } \\
\text { Range }\end{array}$} & \multirow[t]{2}{*}{$\begin{array}{r}\text { No. of } \\
\text { Data }\end{array}$} & \multicolumn{3}{|c|}{ Applied Voltage } & \multicolumn{4}{|c|}{ Radiation Conductance } \\
\hline & & & $\underline{\text { Min }}$ & $\underline{\text { Max }}$ & $\mathbf{U}_{\mathbf{v}}$ & $\underline{\text { Min }}$ & $\underline{\operatorname{Max}}$ & Avg. & $\mathbf{U}_{\mathbf{b}}$ \\
\hline & & & $\mathbf{V}$ & $\mathbf{V}$ & $\pm \%$ & $\overline{\mu S}$ & $\mu \mathrm{S}$ & $\mu \mathrm{S}$ & $\pm \%$ \\
\hline \multirow[t]{4}{*}{$\mathbf{R} 2.1^{\mathbf{k}, \mathbf{n}}$} & low & 2 & 30 & 40 & $\mathbf{i}$ & 10.6 & 10.9 & 10.7 & 17 \\
\hline & med & $\mathbf{0}$ & - & 一 & - & - & 一 & - & - \\
\hline & high & 0 & 一 & - & - & 一 & - & - & - \\
\hline & overall & 2 & 30 & 40 & & 10.6 & 10.9 & 10.7 & \\
\hline \multirow[t]{5}{*}{$\mathrm{R} 2.2^{\mathrm{k}, \mathrm{n}}$} & low & 1 & 18 & 18 & $\mathbf{i}$ & 12.1 & 12.1 & 12.1 & 17 \\
\hline & med & 0 & - & - & - & - & - & - & - \\
\hline & high & 0 & 一 & - & 一 & - & - & - & 一 \\
\hline & overall & 1 & 18 & 18 & & 12.1 & 12.1 & 12.1 & \\
\hline & & & \multicolumn{3}{|c|}{ weighted mean ${ }^{P}$} & & & 11.12 & \\
\hline
\end{tabular}

Table 4. Transfer standard No. 3-20 nominal frequency $5 \mathrm{MHz}$.

\begin{tabular}{|c|c|c|c|c|c|c|c|c|c|}
\hline \multirow[t]{3}{*}{$\operatorname{Code}^{\mathfrak{a}}$} & \multirow[t]{3}{*}{$\begin{array}{l}\text { Power } \\
\text { Range }\end{array}$} & \multirow[t]{3}{*}{$\begin{array}{r}\text { No. of } \\
\text { Data }\end{array}$} & \multicolumn{3}{|c|}{ Applied Voltage } & \multicolumn{4}{|c|}{ Radiation Conductance } \\
\hline & & & Min & $\underline{\text { Max }}$ & $\underline{\mathbf{U}_{\mathbf{V}}}$ & Min & Max & Avg. & $\mathbf{U}_{\mathbf{b}}$ \\
\hline & & & $\mathbf{V}$ & V & $\pm \%$ & $\mu S$ & $\mu \mathrm{S}$ & $\mu S$ & $\pm \%$ \\
\hline \multirow[t]{4}{*}{ RD-1 } & low & 5 & 12 & 12 & 0.25 & 49 & 67 & 58 & 22 \\
\hline & med & 5 & 40 & 40 & 0.25 & 78 & 81 & 78 & 7 \\
\hline & high & 5 & 100 & 100 & 0.25 & 69 & 69 & 69 & 5 \\
\hline & overall & 15 & 12 & 100 & - & 49 & 81 & 68.4 & 一 \\
\hline \multirow[t]{4}{*}{ RD-2 } & low & 0 & - & - & 一 & 一 & - & - & - \\
\hline & med & 6 & 35 & 57 & 1.3 & 69.9 & 72.8 & 71.1 & 10 \\
\hline & high & 0 & - & - & - & - & - & - & - \\
\hline & overall & 6 & 35 & 57 & 1.3 & 69.9 & 72.8 & 71.1 & 10 \\
\hline \multirow[t]{4}{*}{ RD-3 } & low & 10 & 12 & 12 & 0.5 & 54.9 & 110.7 & 93.7 & $46^{c}$ \\
\hline & med & 10 & 40 & 40 & 0.5 & 79.6 & 89.6 & 82.1 & $6.5^{\mathrm{c}}$ \\
\hline & high & 10 & 124 & 126 & 0.5 & 72.0 & 74.6 & 73.6 & 1.6 \\
\hline & overall & 30 & 12 & 126 & - & 54.9 & 110.7 & 83.1 & - \\
\hline \multirow[t]{4}{*}{ RD-4 } & low & $\mathbf{1}$ & $18^{e}$ & 18 & 2.0 & 76.1 & 76.1 & 76.1 & 19 \\
\hline & med & 2 & 35 & 42 & $1.8^{\mathrm{c}}$ & 67.5 & 71.8 & 69.7 & $12.5^{c}$ \\
\hline & high & 0 & - & - & - & - & - & - & - \\
\hline & overall & 3 & 18 & 42 & 一 & 67.5 & 76.1 & 71.8 & - \\
\hline \multirow[t]{4}{*}{ RF-1 } & low & 9 & 10 & 10 & 0.2 & 67.4 & 71.7 & 68.0 & $7.5^{\mathrm{c}, \mathrm{f}}$ \\
\hline & med & 11 & 32 & 33 & 0.2 & 68.3 & 70.4 & 68.8 & $7.4^{\mathrm{c}, \mathrm{f}}$ \\
\hline & high & 9 & 101 & 101 & 0.2 & 70.0 & 72.4 & 70.5 & $7.2^{c, f}$ \\
\hline & overall & 29 & 10 & 101 & - & 67.4 & 72.4 & 69.06 & - \\
\hline \multirow[t]{4}{*}{ RF-3 } & low & 0 & - & - & - & - & - & - & - \\
\hline & med & 12 & 35 & 57 & 1.3 & 69.3 & 73.6 & 72.0 & 10 \\
\hline & high & 6 & 70 & 71 & 1.3 & 69.2 & 69.8 & 69.5 & 10 \\
\hline & overall & 18 & 35 & 71 & - & 69.2 & 73.6 & 71.14 & - \\
\hline \multirow[t]{4}{*}{$\mathrm{RF}-4$} & low & $11 / 24$ & 9 & 14 & 0.41 & 66.1 & 72.8 & 69.4 & 4.5 \\
\hline & med & $12 / 26$ & 36 & 48 & 0.25 & 67.2 & 71.6 & 69.3 & 4.1 \\
\hline & high & $12 / 26$ & 68 & 87 & 0.59 & 67.8 & 71.9 & 70.2 & 4.8 \\
\hline & overall & $35 / 76$ & 9 & 87 & - & 66.1 & 72.8 & 69.7 & - \\
\hline
\end{tabular}


(Continuation) TABLE 4. Transfer standard No. 3-20 nominal frequency $5 \mathrm{MHz}$.

\begin{tabular}{|c|c|c|c|c|c|c|c|c|c|}
\hline \multirow[t]{3}{*}{ Code $^{a}$} & \multirow[t]{3}{*}{$\begin{array}{l}\text { Power } \\
\text { Range }\end{array}$} & \multirow[t]{3}{*}{$\begin{array}{r}\text { No. of } \\
\text { Data }\end{array}$} & \multicolumn{3}{|c|}{ Applied Voltage } & \multicolumn{4}{|c|}{ Radiation Conductance } \\
\hline & & & Min & $\underline{\text { Max }}$ & $\mathrm{U}_{\mathrm{V}}$ & Min & Max & Avg. & $\mathrm{U}_{\mathrm{b}}$ \\
\hline & & & $\mathrm{V}$ & $\mathbf{v}$ & $\pm \%$ & $\mu S$ & $\mu \mathrm{S}$ & $\mu \mathrm{S}$ & $\pm \%$ \\
\hline \multirow[t]{4}{*}{ RM-1 } & low & 25 & 7 & 19 & 0.2 & 69.3 & 70.8 & 70.4 & 2.8 \\
\hline & med & 16 & 20 & 58 & 0.2 & 70.3 & 70.7 & 70.5 & 2.8 \\
\hline & high & 17 & 61 & 112 & 0.2 & 69.3 & 71.0 & 70.7 & 2.8 \\
\hline & overall & 58 & 7 & 112 & - & 69.3 & 71.0 & 70.5 & - \\
\hline \multirow[t]{4}{*}{ C-5 } & low & 3 & 14 & 14 & 1.3 & 71.5 & 75.7 & 74.3 & 10 \\
\hline & med & 6 & 35 & 62 & $1: 3$ & 66.5 & 70.7 & 69.5 & 10 \\
\hline & high & 0 & - & - & - & - & - & - & - \\
\hline & overall & 9 & 14 & 62 & - & 66.5 & 75.7 & 71.1 & - \\
\hline $\mathrm{T}-1$ & $\mathbf{j}$ & 1 & $\mathbf{j}$ & $\mathbf{j}$ & na & na & na & 69.9 & 1 \\
\hline Q-4 & $\mathbf{j}$ & 10 & $\mathbf{j}$ & $\mathbf{j}$ & $\mathbf{i}$ & $\mathbf{i}$ & $\mathbf{i}$ & 64.5 & i \\
\hline \multirow{5}{*}{$R-2^{k}$} & low & 6 & 12 & 16 & $\mathbf{i}$ & 67 & 69 & 68 & 17 \\
\hline & med & 0 & - & - & - & - & - & - & - \\
\hline & high & 0 & - & - & - & - & - & - & - \\
\hline & overall & 6 & 12 & 16 & - & 67 & 69 & 68 & \\
\hline & & & \multicolumn{3}{|c|}{ weighted mean ${ }^{p}$} & & & 70.13 & \\
\hline
\end{tabular}

TABLE 5. Transfer standard No. 3-22 nominal frequency $5 \mathrm{MHz}$.

\begin{tabular}{|c|c|c|c|c|c|c|c|c|c|}
\hline \multirow[t]{2}{*}{$\mathrm{Code}^{\mathrm{a}}$} & \multirow[t]{2}{*}{$\begin{array}{l}\text { Power }^{b} \\
\text { Range }\end{array}$} & \multirow[t]{2}{*}{$\begin{array}{l}\text { No. of } \\
\text { Data }\end{array}$} & \multicolumn{3}{|c|}{ Applied Voltage } & \multicolumn{4}{|c|}{ Radiation Conductance } \\
\hline & & & Min & Max & $\mathbf{U}_{\mathbf{V}}$ & Min & $\operatorname{Max}$ & Avg. & $\mathbf{U}_{b}$ \\
\hline & & & V & $\mathbf{v}$ & $\pm \%$ & $\mu \mathrm{S}$ & $\mu \mathrm{S}$ & $\mu \mathrm{S}$ & $\pm \%$ \\
\hline \multirow[t]{4}{*}{ RD-1 } & low & 5 & 12 & 12 & 0.25 & 69 & 97 & 75 & 22 \\
\hline & med & 5 & 40 & 40 & 0.25 & 77 & 80 & 78 & 7 \\
\hline & high & 5 & 100 & 100 & 0.25 & 69.1 & 69.1 & 69.1 & 5 \\
\hline & overall & 15 & 12 & 100 & - & 69 & 97 & 74.2 & - \\
\hline \multirow[t]{4}{*}{ RD-2 } & low & 0 & - & - & - & - & - & - & - \\
\hline & med & 6 & 35 & 57 & 1.3 & 70.1 & 72.8 & 72.1 & 10 \\
\hline & high & 0 & - & - & - & - & - & - & - \\
\hline & overall & 6 & 35 & 57 & 1.3 & 70.1 & 72.8 & 72.1 & \\
\hline \multirow[t]{4}{*}{ RD-3 } & low & 10 & 12 & 12 & 0.5 & 63.2 & 126.3 & 119.7 & $62^{\mathrm{c}}$ \\
\hline & med & 10 & 40 & 40 & 0.5 & 75.6 & 93.1 & 80.9 & $6.6^{\mathrm{c}}$ \\
\hline & high & 10 & 124 & 126 & 0.5 & 85.8 & 89.9 & 87.9 & 1.5 \\
\hline & overall & 30 & 12 & 126 & - & 63.2 & 126.3 & $96.2^{\circ}$ & - \\
\hline \multirow[t]{4}{*}{ RD-4 } & low & 1 & $18^{e}$ & 18 & 2.0 & 71.7 & 71.7 & 71.7 & 20 \\
\hline & med & 2 & 35 & 42 & 1.8 & 66.0 & 71.8 & 68.9 & 13 \\
\hline & high & 0 & - & - & - & - & - & - & - \\
\hline & overall & 3 & 18 & 42 & - & 66.0 & 71.8 & 69.8 & - \\
\hline
\end{tabular}


(Continuation) TABLE 5. Transfer standard No. 3-22 nominal frequency $5 \mathrm{MHz}$.

\begin{tabular}{|c|c|c|c|c|c|c|c|c|c|}
\hline \multirow[t]{2}{*}{$\operatorname{Code}^{a}$} & \multirow[t]{2}{*}{$\begin{array}{l}\text { Power } \\
\text { Range }\end{array}$} & \multirow[t]{2}{*}{$\begin{array}{l}\text { No. of } \\
\text { Data }\end{array}$} & \multicolumn{3}{|c|}{ Applied Voltage } & \multicolumn{4}{|c|}{ Radiation Conductance } \\
\hline & & & Min & Max & $\mathbf{U}_{\mathbf{v}}$ & Min & Max & Avg. & $\mathbf{U}_{b}$ \\
\hline & & & $\mathbf{V}$ & $\mathbf{V}$ & $\pm \%$ & $\mu S$ & $\mu S$ & $\mu \mathrm{S}$ & $\pm \%$ \\
\hline \multirow[t]{4}{*}{ RF-1 } & low & 12 & 10 & 10 & 0.2 & 71.2 & 71.2 & 71.2 & $7^{\mathrm{c}, l}$ \\
\hline & med & 15 & 32 & 33 & 0.2 & 71.6 & 72.6 & 71.7 & $7^{\mathrm{c}, \mathfrak{f}}$ \\
\hline & high & 13 & 101 & 101 & 0.2 & 73.0 & 73.0 & 73.0 & $7.4^{\mathrm{c}, \mathrm{f}}$ \\
\hline & overall & 40 & 10 & 101 & - & 71.2 & 73.0 & 71.97 & - \\
\hline \multirow[t]{4}{*}{ RF-3 } & low & 0 & - & - & - & - & - & - & - \\
\hline & med & 6 & 35 & 56 & 1.3 & 72.7 & 74.5 & 73.6 & 10 \\
\hline & high & 3 & 70 & 70 & 1.3 & 72.4 & 72.5 & 72.5 & 10 \\
\hline & overall & 9 & 35 & 70 & - & 72.4 & 74.5 & 73.22 & - \\
\hline \multirow[t]{4}{*}{$\mathrm{RF}-4$} & low & $17 / 44^{g}$ & 8 & 14 & 0.41 & 68.4 & 78.6 & 72.7 & 4.5 \\
\hline & med & $16 / 42$ & 33 & 49 & 0.25 & 69.4 & 75.1 & 72.3 & 4.1 \\
\hline & high & $17 / 44$ & 65 & 107 & 0.59 & 69.9 & 76.5 & 73.4 & 4.8 \\
\hline & overall & $50 / 130$ & 8 & 107 & - & 68.4 & 78.6 & 72.81 & - \\
\hline \multirow[t]{4}{*}{ RM-1 } & low & 26 & 7 & 18 & 0.2 & 72.0 & 74.3 & 72.8 & 2.8 \\
\hline & med & 16 & 20 & 57 & 0.2 & 72.3 & 73.3 & 72.8 & 2.8 \\
\hline & high & 22 & 61 & 111 & 6.2 & 72.2 & 72.7 & 72.5 & 2.8 \\
\hline & overall & 64 & 7 & 111 & - & 72.0 & 74.3 & 72.68 & - \\
\hline C-3 $3^{\text {h }}$ & i & $\mathbf{i}$ & $\mathrm{i}$ & i & 一 & $\mathbf{i}$ & i & 73 & 25 \\
\hline \multirow[t]{4}{*}{ C-5 } & low & 3 & 14 & 14 & 1.3 & 71.5 & 72.9 & 72.0 & 10 \\
\hline & med & 6 & 35 & 57 & 1.3 & 68.5 & 74.8 & 71.3 & 10 \\
\hline & high & 0 & - & 一 & - & - & 一 & 一 & - \\
\hline & overall & 9 & 14 & 57 & - & 68.5 & 74.8 & 71.52 & - \\
\hline $\mathbf{T}-1$ & $\mathbf{j}$ & 1 & $\mathbf{j}$ & j & na & na & na & 72.5 & 1 \\
\hline$Q-4$ & $\mathbf{j}$ & 10 & $\mathbf{j}$ & $\mathbf{j}$ & $\mathbf{i}$ & $\mathbf{i}$ & $\mathbf{i}$ & 67.6 & $\mathbf{i}$ \\
\hline \multirow[t]{5}{*}{$\mathbf{R}-2^{\mathbf{k}}$} & low & 2 & 12 & 17 & $\mathbf{i}$ & 68 & 69 & 69 & 17 \\
\hline & med & 2 & 32 & 42 & $\mathbf{i}$ & 64 & 71 & 67 & 17 \\
\hline & high & 0 & - & - & - & - & - & - & - \\
\hline & overall & 4 & 12 & 42 & - & 64 & 71 & 68.0 & - \\
\hline & & & \multicolumn{3}{|c|}{ weighted mean ${ }^{p}$} & & & 72.22 & \\
\hline
\end{tabular}

See table 1 .

"See "Procedures and Ingtructions" in Appendix.

The error is not the same for all measurements; the median is given.

dThe average is badly biased by the poor accuracy of the low-power values. If these are disregarded as outliers the average value is I1.45.

-All voltages in this group were reported as peak-to-peak and converted to rms by the pilot laboratory.

'The estimated errors were not symmetrical, that is, the positive values were not quite equal to the negative.

${ }^{8}$ For RF-4, the first number, e.g., 9, is the number of independent groups into which the second number, e.g., 20, which is the number of measurements, is divided. Furthermore, each of the (say) 20 measurements is the average of 4 power readings at the same voltage, two taken as the voltage was switched on, and two as it was switched off.

hystem described as "relatively unproven."

iNot given.

Not given but very low,

kAccording to the investigator, "These results are included for the record but are not regarded as part of the principal measurements."

mSame as (d) but average is 12.25 .

"The investigator considers that the data form two independent groups, here designated-.1 and-.2. He suspects that transducer no. 3-18 auffered a physical change between runs.

'Same as (d) but average is 84.4.

PCalculated from the "overall" values and their ranges by the method given in the text. 
TABLE 6. Deviation of radiation conductance from grand average.

\begin{tabular}{|c|c|c|c|c|c|c|c|c|c|c|c|c|c|c|}
\hline \multirow[t]{3}{*}{$\operatorname{Code}^{a}$} & \multirow[t]{3}{*}{$\begin{array}{l}\text { Power } \\
\text { Range }\end{array}$} & \multirow[t]{3}{*}{$\begin{array}{l}\text { No. of } \\
\text { Data }\end{array}$} & \multicolumn{12}{|c|}{ Deviation, $\left(\bar{G}_{r}-\overline{\bar{G}}_{r}\right) / \bar{G}_{r}$ in percent } \\
\hline & & & \multicolumn{3}{|c|}{ 3-16 } & \multicolumn{3}{|c|}{$3-18$} & \multicolumn{3}{|c|}{$3-20$} & \multicolumn{3}{|c|}{$3-22$} \\
\hline & & & Min & Max & Avg. & Min & Max & Avg. & Min & Max & Avg. & Min & $\operatorname{Max}$ & Avg. \\
\hline RD-1 & $\begin{array}{l}\text { low } \\
\text { med } \\
\text { high } \\
\text { overall }\end{array}$ & $\begin{array}{l}5,5,5,5 \\
5,5,5,5 \\
5,5,5,5 \\
15,15,15,15\end{array}$ & $\begin{array}{c}-21 \\
0.5 \\
4.9 \\
-21\end{array}$ & $\begin{array}{l}4.9 \\
4.0 \\
6.6 \\
6.6\end{array}$ & $\begin{array}{r}-3.8 \\
1.4 \\
5.8 \\
0.9\end{array}$ & $\begin{array}{l}-28 \\
-0.2 \\
5.2 \\
-28\end{array}$ & $\begin{array}{r}-10 \\
0.7 \\
7.0 \\
7.0\end{array}$ & $\begin{array}{r}-19 \\
0.7 \\
-6.1 \\
-4.1\end{array}$ & $\begin{array}{c}-30 \\
11 \\
-1.6 \\
-30\end{array}$ & $\begin{array}{l}-4.5 \\
15 \\
-1.6 \\
15\end{array}$ & $\begin{array}{c}-17 \\
11 \\
-1.6 \\
-2.5\end{array}$ & $\begin{array}{r}-4.5 \\
6.6 \\
-4.3 \\
-4.5\end{array}$ & $\begin{array}{l}34 \\
11 \\
-4.3 \\
34\end{array}$ & $\begin{array}{c}3.8 \\
8.0 \\
-4.3 \\
2.7\end{array}$ \\
\hline RD-2 & $\begin{array}{l}\text { low } \\
\text { med } \\
\text { high } \\
\text { overall }\end{array}$ & $\begin{array}{l}3,3,0,0 \\
12,12,6,6 \\
0,0,0,0 \\
15,15,6,6\end{array}$ & $\begin{array}{l}2.3 \\
-2.1 \\
-2.1\end{array}$ & $\begin{array}{l}3.1 \\
4.0 \\
- \\
4.0\end{array}$ & $\begin{array}{l}3.1 \\
0.5 \\
\overrightarrow{1} .2\end{array}$ & $\begin{array}{l}6.1 \\
0.7 \\
- \\
0.7\end{array}$ & $\begin{array}{c}7.0 \\
6.1 \\
-7.0\end{array}$ & $\begin{array}{l}7.0 \\
3.4 \\
- \\
4.0\end{array}$ & $\begin{array}{l}\overline{-0.3} \\
\overline{-3.0}\end{array}$ & $\begin{array}{l}- \\
3.8 \\
\overline{3.8}\end{array}$ & $\begin{array}{l}\overline{1.4} \\
\overline{1.4}\end{array}$ & $\begin{array}{l}\overline{-2.9} \\
\overline{-2.9}\end{array}$ & $\begin{array}{l}\overline{0.8} \\
\overline{0.8}\end{array}$ & $\begin{array}{c}\overline{-} \\
-0.2 \\
-\overline{0.2}\end{array}$ \\
\hline RD-3 & $\begin{array}{l}\text { low } \\
\text { med } \\
\text { high } \\
\text { overall }\end{array}$ & $\begin{array}{l}10,10,10,10 \\
10,10,10,10 \\
10,10,10,10 \\
30,30,30,30\end{array}$ & $\begin{array}{r}118 \\
-9.1 \\
0.5 \\
-9.1\end{array}$ & $\begin{array}{c}237 \\
10.1 \\
10.1 \\
237\end{array}$ & $\begin{array}{c}161 \\
-4.7 \\
4.9 \\
54^{\mathrm{d}}\end{array}$ & $\begin{array}{c}47 \\
13 \\
0.7 \\
0.7\end{array}$ & $\begin{array}{c}287 \\
26 \\
3.4 \\
287\end{array}$ & $\begin{array}{c}182 \\
19 \\
1.6 \\
\mathrm{rg}^{\mathrm{m}}\end{array}$ & $\begin{array}{c}-22 \\
13 \\
2.7 \\
-22\end{array}$ & $\begin{array}{l}58 \\
28 \\
6.4 \\
58\end{array}$ & $\begin{array}{l}34 \\
17 \\
4.9 \\
18\end{array}$ & $\begin{array}{c}-12 \\
4.7 \\
19 \\
-12\end{array}$ & $\begin{array}{l}75 \\
29 \\
24 \\
75\end{array}$ & $\begin{array}{l}66 \\
12 \\
22 \\
33\end{array}$ \\
\hline RD-4 & $\begin{array}{l}\text { low } \\
\text { med } \\
\text { high } \\
\text { overall }\end{array}$ & $\begin{array}{l}1,1,1,1 \\
2,2,2,2 \\
0,0,0,0 \\
3,3,3,3\end{array}$ & $\begin{array}{l}-19 \\
-5.6 \\
-19\end{array}$ & $\begin{array}{c}-19 \\
2.3 \\
- \\
2.3\end{array}$ & $\begin{array}{l}-19 \\
-2.1 \\
-7.3\end{array}$ & $\begin{array}{l}-8.3 \\
5.6 \\
-8.3\end{array}$ & $\begin{array}{c}-8.3 \\
2.5 \\
- \\
2.5\end{array}$ & $\begin{array}{r}.3 \\
1 \\
-3.8\end{array}$ & $\begin{array}{l}8.5 \\
-3.7 \\
- \\
-3.7\end{array}$ & $\begin{array}{l}8.5 \\
2.4 \\
\overline{8.5}\end{array}$ & $\begin{array}{c}8.5 \\
-0.6 \\
- \\
2.4\end{array}$ & $\begin{array}{l}-0.7 \\
-8.6 \\
- \\
-8.6\end{array}$ & $\begin{array}{c}-0.7 \\
-0.6 \\
-0.6\end{array}$ & $\begin{array}{l}-0.7 \\
-4.6 \\
- \\
-3.4\end{array}$ \\
\hline RF-1 & $\begin{array}{l}\text { low } \\
\text { med } \\
\text { high } \\
\text { overall }\end{array}$ & $\begin{array}{l}6,7,9,12 \\
6,7,11,15 \\
7,7,9,13 \\
19,21,29,40\end{array}$ & $\begin{array}{l}-7.3 \\
-4.7 \\
-3.0 \\
-7.3\end{array}$ & $\begin{array}{r}-2.7 \\
-2.1 \\
3.1 \\
3.1\end{array}$ & $\begin{array}{l}-4.7 \\
-3.8 \\
-0.3 \\
-2.7\end{array}$ & $\begin{array}{l}-10 \\
-10 \\
-5.6 \\
-10\end{array}$ & $\begin{array}{l}-2.9 \\
-3.8 \\
-0.2 \\
-0.2\end{array}$ & $\begin{array}{l}-6.5 \\
-7.4 \\
-3.8 \\
-6.0\end{array}$ & $\begin{array}{l}-3.9 \\
-2.6 \\
-0.2 \\
-3.9\end{array}$ & $\begin{array}{l}2.2 \\
0.4 \\
3.2 \\
3.2\end{array}$ & $\begin{array}{r}-3.0 \\
-1.9 \\
0.5 \\
-1.5\end{array}$ & $\begin{array}{r}-1.4 \\
-0.9 \\
1.1 \\
-1.4\end{array}$ & $\begin{array}{r}-1.4 \\
0.5 \\
1.1 \\
1.1\end{array}$ & $\begin{array}{r}-1.4 \\
-0.7 \\
1.1 \\
-0.3\end{array}$ \\
\hline RF-3 & $\begin{array}{l}\text { low } \\
\text { med } \\
\text { high } \\
\text { overall }\end{array}$ & $\begin{array}{l}3,3,0,0 \\
6,6,12,6 \\
0,0,6,3 \\
9,9,18,9\end{array}$ & $\begin{array}{l}2.3 \\
4.0 \\
- \\
2.3\end{array}$ & $\begin{array}{l}3.1 \\
4.9 \\
- \\
4.9\end{array}$ & $\begin{array}{l}2.3 \\
4.0 \\
- \\
3.7\end{array}$ & $\begin{array}{l}-1.1 \\
2.5 \\
-1.1\end{array}$ & $\begin{array}{l}1.6 \\
3.4 \\
- \\
3.4\end{array}$ & $\begin{array}{l}0.7 \\
2.5 \\
- \\
2.1\end{array}$ & $\begin{array}{l}\overline{-1.2} \\
-1.3 \\
-1.3\end{array}$ & $\begin{array}{r}- \\
4.9 \\
-0.5 \\
4.9\end{array}$ & $\begin{array}{c}- \\
2.7 \\
-0.9 \\
1.4\end{array}$ & $\begin{array}{l}-\overline{0.7} \\
0.2 \\
0.2\end{array}$ & $\begin{array}{l}- \\
3.2 \\
0.4 \\
3.2\end{array}$ & $\begin{array}{l}- \\
1.9 \\
0.4 \\
1.4\end{array}$ \\
\hline $\mathrm{RF}-4$ & $\begin{array}{l}\text { low } \\
\text { med } \\
\text { high } \\
\text { overall }\end{array}$ & $\begin{array}{l}20,0,24,44 \\
20,0,26,42 \\
20,0,26,44 \\
60,0,76,130\end{array}$ & $\begin{array}{l}-10 \\
-9.1 \\
-8.2 \\
-10\end{array}$ & $\begin{array}{l}-2.1 \\
-1.2 \\
-2.1 \\
-1.2\end{array}$ & $\begin{array}{l}-4.7 \\
-4.7 \\
-4.7 \\
-5.0\end{array}$ & $\begin{array}{l}- \\
- \\
-\end{array}$ & $\begin{array}{l}- \\
- \\
-\end{array}$ & $\begin{array}{l}- \\
- \\
-\end{array}$ & $\begin{array}{l}-5.7 \\
-4.2 \\
-3.3 \\
-5.7\end{array}$ & $\begin{array}{l}3.8 \\
2.1 \\
2.5 \\
3.8\end{array}$ & $\begin{array}{r}-1.0 \\
-1.2 \\
0.1 \\
-0.6\end{array}$ & $\begin{array}{l}-5.3 \\
-3.9 \\
-3.2 \\
-5.3\end{array}$ & $\begin{array}{l}8.8 \\
4.0 \\
5.9 \\
8.8\end{array}$ & $\begin{array}{l}0.7 \\
0.1 \\
1.6 \\
0.8\end{array}$ \\
\hline $\mathrm{RF}-4.1^{\mathrm{n}}$ & $\begin{array}{l}\text { low } \\
\text { med } \\
\text { high } \\
\text { overall }\end{array}$ & $\begin{array}{l}0,26,0,0 \\
0,26,0,0 \\
0,22,0,0 \\
0,74,0,0\end{array}$ & $\begin{array}{l}- \\
- \\
-\end{array}$ & $\begin{array}{l}- \\
- \\
-\end{array}$ & $\begin{array}{l}- \\
- \\
-\end{array}$ & $\begin{array}{r}-0.7 \\
-0.2 \\
6.1 \\
-0.2\end{array}$ & $\begin{array}{l}12 \\
5.2 \\
9.7 \\
12\end{array}$ & $\begin{array}{l}5.2 \\
2.5 \\
7.0 \\
4.8\end{array}$ & $\begin{array}{l}- \\
- \\
-\end{array}$ & $\begin{array}{l}- \\
- \\
-\end{array}$ & $\begin{array}{l}- \\
- \\
-\end{array}$ & $\begin{array}{l}- \\
- \\
-\end{array}$ & $\begin{array}{l}- \\
- \\
-\end{array}$ & $\begin{array}{l}- \\
- \\
-\end{array}$ \\
\hline $\mathrm{RF}-4.2^{\mathrm{n}}$ & $\begin{array}{l}\text { low } \\
\text { med } \\
\text { high } \\
\text { overall }\end{array}$ & $\begin{array}{l}0,20,0,0 \\
0,20,0,0 \\
0,20,0,0 \\
0,60,0,0\end{array}$ & $\begin{array}{l}- \\
- \\
-\end{array}$ & $\begin{array}{l}- \\
- \\
-\end{array}$ & $\begin{array}{l}- \\
- \\
-\end{array}$ & $\begin{array}{l}-7.4 \\
-7.4 \\
-6.5 \\
-7.4\end{array}$ & $\begin{array}{l}-1.1 \\
-2.0 \\
-2.0 \\
-1.1\end{array}$ & $\begin{array}{l}-3.8 \\
-4.7 \\
-3.8 \\
-4.2\end{array}$ & $\begin{array}{l}- \\
- \\
-\end{array}$ & $\begin{array}{l}- \\
- \\
-\end{array}$ & $\begin{array}{l}- \\
- \\
-\end{array}$ & $\begin{array}{l}- \\
- \\
-\end{array}$ & $\begin{array}{l}- \\
- \\
-\end{array}$ & $\begin{array}{l}- \\
- \\
-\end{array}$ \\
\hline RM-I & $\begin{array}{l}\text { low } \\
\text { med } \\
\text { high } \\
\text { overall }\end{array}$ & $\begin{array}{l}34,32,25,26 \\
15,13,16,16 \\
17,19,17,22 \\
66,64,58,64\end{array}$ & $\begin{array}{l}-2.1 \\
-1.2 \\
-1.2 \\
-2.1\end{array}$ & $\begin{array}{r}0.5 \\
-1.2 \\
-1.2 \\
0.5\end{array}$ & $\begin{array}{l}-0.3 \\
-1.2 \\
-1.2 \\
-0.9\end{array}$ & $\begin{array}{l}-1.1 \\
-1.1 \\
-1.1 \\
-1.1\end{array}$ & $\begin{array}{l}1.6 \\
0.7 \\
2.5 \\
2.5\end{array}$ & $\begin{array}{r}0.7 \\
-0.2 \\
0.7 \\
0.4\end{array}$ & $\begin{array}{r}-1.2 \\
0.2 \\
0.1 \\
-1.2\end{array}$ & $\begin{array}{l}1.0 \\
0.8 \\
1.2 \\
1.2\end{array}$ & $\begin{array}{l}0.4 \\
0.5 \\
0.8 \\
0.5\end{array}$ & $\begin{array}{r}-0.3 \\
0.1 \\
0.0 \\
-0.3\end{array}$ & $\begin{array}{l}2.9 \\
1.5 \\
0.7 \\
2.9\end{array}$ & $\begin{array}{l}0.8 \\
0.8 \\
0.4 \\
0.6\end{array}$ \\
\hline C-3 & $\mathbf{h}$ & $\mathbf{i}, \mathbf{i}, \mathbf{i}, \mathbf{i}$ & $\mathbf{i}$ & i & -3.0 & - & - & - & - & - & - & $\mathbf{i}$ & $\mathbf{i}$ & 1.1 \\
\hline C-5 & $\begin{array}{l}\text { low } \\
\text { med } \\
\text { high } \\
\text { overall }\end{array}$ & $\begin{array}{l}3,3,3,3 \\
3,3,6,6 \\
0,0,0,0 \\
6,6,9,9\end{array}$ & $\begin{array}{c}-1.2 \\
3.1 \\
-1.2\end{array}$ & $\begin{array}{l}7.5 \\
8.4 \\
8.4\end{array}$ & $\begin{array}{l}3.1 \\
5.8 \\
4.4\end{array}$ & $\begin{array}{l}1.6 \\
-2.0 \\
-2.0\end{array}$ & $\begin{array}{l}7.0 \\
9.7 \\
\overline{9.7}\end{array}$ & $\begin{array}{l}3.4 \\
3.4 \\
- \\
3.4\end{array}$ & $\begin{array}{l}2.0 \\
-5.2 \\
-5.2\end{array}$ & $\begin{array}{l}7.9 \\
0.8 \\
7.9\end{array}$ & $\begin{array}{c}5.9 \\
-0.9 \\
- \\
1.4\end{array}$ & $\begin{array}{l}-1.0 \\
-5.2 \\
-5.2\end{array}$ & $\begin{array}{l}0.9 \\
3.6 \\
- \\
3.6\end{array}$ & $\begin{array}{l}-0.3 \\
-1.3 \\
-\overline{-1.0}\end{array}$ \\
\hline
\end{tabular}




\begin{tabular}{|c|c|c|c|c|c|c|c|c|c|c|c|c|c|c|}
\hline \multirow[t]{3}{*}{$\operatorname{Code}^{\mathrm{a}}$} & \multirow[t]{3}{*}{$\begin{array}{l}\text { Power } \\
\text { Range }\end{array}$} & \multirow[t]{3}{*}{$\begin{array}{l}\text { No. of } \\
\text { Data }\end{array}$} & \multicolumn{12}{|c|}{ Deviation, $\left(\bar{G}_{\tau}-\overline{\mathbf{G}}_{\mathrm{r}}\right) / \overline{\mathbf{G}}_{\mathrm{r}}$ in percent } \\
\hline & & & \multicolumn{3}{|c|}{$3-16$} & \multicolumn{3}{|c|}{$3-18$} & \multicolumn{3}{|c|}{$3-20$} & \multicolumn{3}{|c|}{ 3-22 } \\
\hline & & & Min & Max & Avg. & Min & Max & Avg. & Min & Max & Avg. & Min & Max & Avg. \\
\hline \multirow[t]{4}{*}{$0-1$} & low & $0,0,0,0$ & - & - & - & - & - & - & - & - & - & - & - & - \\
\hline & med & $9,9,0,0$ & -7.3 & 5.8 & -1.2 & -2.9 & 7.0 & 2.5 & - & - & - & - & - & - \\
\hline & high & $5,6,0,0$ & -4.7 & 2.3 & -1.2 & -2.9 & 3.4 & 1.6 & - & - & - & - & - & - \\
\hline & overall & $14,15,0,0$ & -7.3 & 5.8 & -1.3 & -2.9 & 7.0 & 1.9 & - & - & - & - & - & - \\
\hline T-1 & & $0,0,1,1$ & - & - & - & - & - & - & na & na & -0.3 & na & na & 0.4 \\
\hline Q-4 & & $10,10,10,10$ & $\mathbf{i}$ & $\mathbf{i}$ & -7.7 & $\mathbf{i}$ & $\mathbf{i}$ & -7.9 & $\mathbf{i}$ & $\mathbf{i}$ & -8.0 & $\mathbf{i}$ & $\mathbf{i}$ & -6.4 \\
\hline \multirow[t]{4}{*}{$R-2^{k}$} & low & $6,0,6,2$ & -4.7 & 0.5 & -3.0 & - & - & - & -4.5 & -1.6 & -3.0 & -5.8 & -4.5 & -4.5 \\
\hline & med & $0,0,0,2$ & - & - & - & - & - & - & - & - & - & -11 & -1.7 & -7.2 \\
\hline & high & $0,0,0,0$ & - & - & - & - & - & - & - & - & - & - & - & - \\
\hline & overall & $6,0,6,4$ & -4.7 & 0.5 & $-\mathbf{3 . 0}$ & - & - & - & -4.5 & -1.6 & -3.0 & -11 & -1.7 & -5.8 \\
\hline \multirow[t]{4}{*}{ R-2.1 } & low & $0,2,0,0$ & - & - & - & -4.7 & -2.0 & -3.8 & - & - & - & - & - & - \\
\hline & med & $0,0,0,0$ & - & - & - & - & - & - & - & - & - & - & - & - \\
\hline & high & $0,0,0,0$ & - & - & - & - & - & - & - & - & - & - & - & - \\
\hline & overall & $0,2,0,0$ & - & - & - & -4.7 & -2.0 & -3.8 & - & - & - & - & - & - \\
\hline \multirow[t]{4}{*}{ R-2.2 } & low & $0,1,0,0$ & - & - & - & 8.8 & 8.8 & 8.8 & - & - & - & - & - & - \\
\hline & med & $0,0,0,0$ & - & - & - & - & - & - & - & - & - & - & - & - \\
\hline & high & $0,0,0,0$ & - & - & - & - & - & - & - & - & - & - & - & - \\
\hline & overall & $0,1,0,0$ & - & - & - & 8.8 & 8.8 & 8.8 & - & - & - & - & - & - \\
\hline
\end{tabular}

a,b,k, h, and nsee footnotes to tables $2,3,4$, and 5 .

cFor $3-16,18,20$, and 22 respectively.

dThis average is badly biased by the poor accuracy of the low-power values. If these are disregarded as outliers, the value becomes $-0.1 \%$.

mSame as d but value becomes $10 \%$.

'Same as ${ }^{\mathrm{d}}$ but value is $17 \%$.

TABle 7. Cumulative distribution of the deviations of tables $2,3,4$, and 5 .

\begin{tabular}{|c|c|c|c|c|}
\hline \multirow[b]{2}{*}{ Frequency, MHz } & \multicolumn{4}{|c|}{ No. of cases for which $\left(\bar{G}_{r}-\bar{G}_{r}\right) / \bar{G}_{r}<\Delta$} \\
\hline & & 2 & & 5 \\
\hline$\Delta$ & No. & $\begin{array}{l}\text { Fraction } \\
\text { of total } \\
\end{array}$ & №. & $\begin{array}{c}\text { Fraction } \\
\text { of total }\end{array}$ \\
\hline$\%$ & & $\%$ & & $\%$ \\
\hline 1 & 3 & 11 & 10 & 40 \\
\hline 2 & 6 & 22 & 17 & 68 \\
\hline 3 & 9 & 33 & 20 & 80 \\
\hline 4 & 14 & 52 & 21 & 84 \\
\hline 5 & 19 & 70 & 21 & 84 \\
\hline 6 & 20 & 74 & 22 & 88 \\
\hline 7 & 21 & 78 & 23 & 92 \\
\hline 8 & 24 & 89 & 23 & 92 \\
\hline 9 & 25 & 93 & 23 & 92 \\
\hline$\infty$ & 27 & 100 & 25 & 100 \\
\hline
\end{tabular}

(although a 3- rather than a 2-MHz unit) exhibited a large shift in the measured $G_{r}$. Upon autopsy it was found to have suffered a small crack in the epoxy seal and therefore a small leak. Upon being dried out and recemented the transducer yielded its original $G_{r}$.

One could speculate that a small leak occurred while transducer 3-18 was at the participant's laboratory and that on the way back to the pilot laboratory the transducer dried out. Sixteen months later, when the last measurements were made, the transducer was still behaving properly, at least under the pilot laboratory conditions. While we were at it, we took additional measurements on the other transducers as well. All of the measurements made by the modulation radiationpressure method at the pilot laboratory are summarized in table 8.

From the material in table 8 , we could conclude that whatever drifts occur in either the transducers or the ap- 
paratus itself are of no great consequence. However, close examination, using standard statistical tests, shows that the disparities are not entirely random. Indeed, the regressions of $G_{r}$ on time show trends which are significant although not overwhelmingly so. To elucidate this question will require a carefully planned experimental design carried out over several years, and this is under serious consideration.

TABLe 8. Summary of radiation conductance, $G_{r}$.

\begin{tabular}{lccccc}
\hline \hline & \multicolumn{2}{c}{$2 \mathrm{MHz}$} & & \multicolumn{2}{c}{$5 \mathrm{MHz}$} \\
\cline { 2 - 3 } \cline { 5 - 6 } & & & & \\
\hline Transducer & $3-16$ & $3-18$ & $3-20$ & $3-22$ \\
n (over about 1233 days) & 87 & 85 & 79 & 85 \\
$\overline{\mathrm{G}}_{\mathrm{r}}$ (Grand average) $\mu \mathrm{S}$ & 11.352 & 11.150 & 70.42 & 72.36 \\
$\begin{array}{c}\text { Standard deviation } \\
\mu \mathrm{S}\end{array}$ & & & & \\
\multicolumn{1}{c}{$\%$} & 0.075 & 0.106 & 0.21 & 0.40 \\
& 0.51 & 0.95 & 0.31 & 0.55 \\
\hline
\end{tabular}

\section{References}

[1] Zapf, Thomas L. Calibration of quartz transducers as ultrasonic power standards by an electrical method. IEEE Ultrasonics Symposium Proceedings: Cat. \# 74 CHO ISU; 1974; 45-49.

[2] Greenspan, M., Breckenridge, F.R., Tschiegg, C.E. Ultrasonic transducer power output by modulated radiation pressure. $\mathbf{J}$. Acoust. Soc. Am. 63(4): 1031-1038; 1978.

[3] Zapf, T.L., Harvey, M.E., Larsen, N.T., Stoltenberg, R.T. Ultrasonic calorimeter for beam power measurements. Natl. Bur. Stand. (U.S.) Tech. Note 686; 1976 September. 36 p.

[4] Snedecor, George W. Statistical methods, 6 ed. Ames: Iowa State University Press; 1967.593 p.

\section{Appendix}

\section{International Infercomparison of cw Ultrasonic Beam Power Measurements - Procedures and Instructions}

$$
\begin{gathered}
\text { National Bureau of Standards } \\
\text { Washington, DC } 20234
\end{gathered}
$$

This document provides instructions and a typical procedure for the intercomparison of ultrasonic beam power measurements. The intercomparison is being conducted by circulating to participating laboratories four transfer standards, namely, half-wave resonant, airbacked, quartz transducers.

\subsection{Equipment Supplied by NBS}

1. Transfer standards. The resonance frequencies of the transducers have been measured at NBS.

2. Matehing circuit. A matching circuit may be needed to provide adequate voltage to the quartz transducers, which have a high electrical input impedance. It also helps to minimize distortion in the voltage applied to the transducers.

3. Connectors. Adapters are supplied for use with the transducers.

\subsection{Care of the Standard Transducers}

1. Avoid any severe mechanical shock.

2. Voltage limitations on the transducers are $3 \mathrm{~V}$ unloaded, and $350 \mathrm{~V}$ water loaded. Transducer voltage should be monitored at all times when connected to a power amplifier. Experimental procedures should be used that will ensure that the test voltage is not applied when the transducer is out of water and that the voltage is removed before removing the transducer from the water.

3. The transducer connectors are not waterproof, and should not be submerged.

4. The front faces of the transducers are gold plated. They should be carefully wiped with lens tissue to remove water. Avoid excessive force.

\subsection{Measurements To Be Made}

The quantity to be measured is the total $\mathrm{cw}$ beam power radiated by a transducer (the supplied transfer standard) into a reflectionless water load at a specified temperature, frequency, and sinusoidal input voltage to the transducer. At the power levels to be used in the intercomparison, the beam power is proportional to the square of the applied voltage. Each participant is responsible for obtaining a suitable if voltmeter and having it calibrated at the voltages and frequencies required. The following table indicates the specified frequency, $f_{s}$, and the specified voltage, $V_{s}$, at which each measurement is to be made. The actual frequency, $f_{m}$, of the measurement should be within $\pm 0.02 \%$ of $f_{s}$. The measured voltage, $V_{m}$, should be within $\pm 50 \%$ of $V_{s}$ (subject to the $350 \mathrm{~V}$ maximum limitaticn). The voltages are arbitrary, but have been chosen to cover the range of interest.

\subsection{Reporting}

Please use the reporting form included herewith. Report the test data in the sequence in which the data 
were taken.

The pilot laboratory will accumulate and summarize the results from all participating laboratories. As discussed in the following paragraphs, numerical results from each participant should be reported in a manner to allow comparison on a common basis.

An error can result from attenuation in the water coupling medium between the transducer and the receiver target. The radiated beam power, $P_{r}$, equals the received power measured by the participant's equipment, $P_{m}$, plus the power loss in the coupling medium. This loss should be determined by measurement or calculation, and applied as a correction as indicated below. To correct for the attenuation, the following formula may be used:

$$
P_{r}=P_{m} \bullet \exp 44 \times 10^{-17} f_{m}^{2} d,
$$

where $d$ is the water path distance in centimeters between the transducer and the point at which the power measurement is made, and $f_{m}$ is the measurement frequency in $\mathrm{Hz}$.

The temperature of the water coupling medium should be as close as possible to $23^{\circ} \mathrm{C}$, and in the range of $20^{\circ} \mathrm{C}$ to $26^{\circ} \mathrm{C}$. A correction to a common-basis temperature and voltage should be applied as follows:

$$
P_{b}=P_{r}\left(V_{s} / V_{m}\right)^{2}\left[1+0.0016\left(T_{m}-23\right)\right],
$$

where $P_{b}$ is the "common basis power" that would be measured at $23{ }^{\circ} \mathrm{C}$ with the common basis voltage, $V_{s}$, applied to the transducer, $V_{m}$ is the measured applied voltage, $T_{m}$ is the measurement temperature in ${ }^{\circ} \mathrm{C}$. The coefficient 0.0016 is related to the temperature dependence of the ec of water.

So that the fractional uncertainty, $U_{b}$, associated with the common-basis power is properly determined at each test point, the participant should estimate the fractional uncertainty, $U_{r}$, in the radiated power, taking into account the uncertainty in measuring or calculating the loss in the coupling medium. For example, if $P_{r}$ is 0.137 watts and the uncertainty in $P_{r}$ is \pm 0.004 watts, then $U_{r}$ $= \pm 0.004 / 0.137= \pm 0.03$, or $\pm 3 \%$. The commonbasis power uncertainty, $U_{b}$, is then calculated as

$$
U_{b}=U_{r}+2 U_{v}
$$

where $U_{v}$ is the fractional uncertainty in the voltmeter calibration and the factor 2 is a result of the square-law relationship between voltage and power. Uncertainties associated with the application of the temperature correction will be negligible if temperatures are kept within the specified range. The estimate of $U_{r}$ should be based on the participant's experience with his equipment; it should not be influenced by the variations observed in the present intercomparison.

Provide a brief description of each set of equipment and method of measurement used in the intercomparison. If more than one method of measurement is used, please report each on a separate data sheet.

If the quantities $V_{m}$ and $P_{m}$ are inappropriate to the method of measurement used, then please supply at least the following information for each measurement: temperature of the measurement, $T_{m}$; frequency of the measurement, $f_{m}$; the radiation conductance, $G_{r}$, measured for the transducer; and the estimated uncertainty, $U_{b}$, of the measured $G_{r}$.

\subsection{Precautions}

The transducer must be oriented so that the entire ultrasonic beam will be received by the participant's measuring equipment. Reflections that would cause ultrasonic energy to return to the transducer must be reduced to a negligible level to prevent interference at the face of the transducer that may change the characteristics of the transfer standard.

Care should be exercised to avoid the presence or formation of bubbles on the transducer face during test. The use of degassed water may eliminate this problem.

\subsection{Typical Procedure}

The following information is included but may be disregarded if not applicable:

\section{Typical equipment provided by participants:}

1. Voltmeter. A radio-frequency voltmeter is needed to measure the voltage applied to the transducers. The voltmeter must be calibrated at the frequencies and voltages listed under Measurements to be made. The voltmeter should be calibrated with a short cable. This will minimize loading on the matching network. This cable then becomes part of the calibrated voltmeter. A calibration uncertainty of $\pm 0.25 \%$ or better is desirable.

2. Power amplifier. A power amplifier capable of supplying 5 watts into a $50-0 h m$ load should be adequate.

3. Signal generator. A stable generator must drive the power amplifier with a cw sinusoidal waveform of low distortion (preferably less than $0.5 \%$ ).

4. Frequency counter. This is needed to set the frequency of the signal applied to the transducer. 


\section{Typical procedure}

1. Interconnect equipment as indicated in figure A-1, but do not turn on the signal generator and power amplifier before first setting the output level controls to minimum. NOTE: CONNECTORS ON THE TRANSDUCERS ARE NOT WATERPROOF AND MUST BE PROTECTED IF IMMERSED IN WATER.

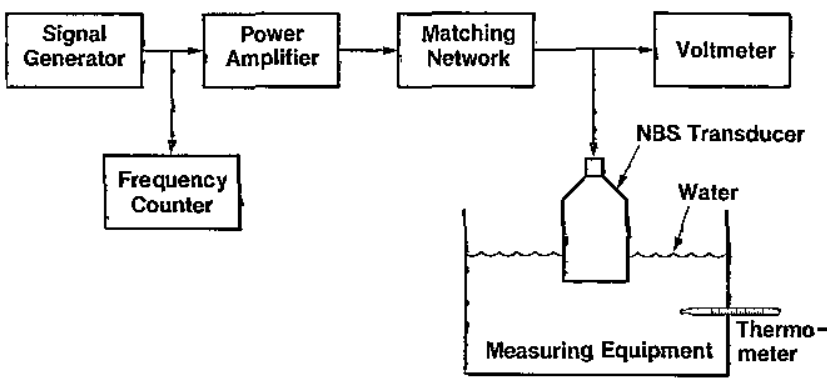

Figure A-1. Example of possible test assembly.

2. Prepare the measuring equipment for measurements of power at a temperature of $23^{\circ} \mathrm{C} \pm 3^{\circ}$.

3. Place the NBS transducer in the water coupling port of the measuring equipment. Brush away any bubbles adhering to the face of the transducer. CAUTION - DO NOT APPLY VOLTAGE TO TRANSDUCER UNLESS THE GOLD TRANSDUCER FACE IS IN THE WATER (i.e., THE TRANSDUCER IS LOADED). AP. PLICATION OF HIGH VOLTAGE TO AN UNLOADED QUARTZ TRANSDUCER (i.e., IN AIR) MAY DAMAGE IT.

4. Set the signal generator controls to obtain a cw sinusoidal waveform. Set the frequency controls of the signal generator approximately to the specified resonance frequency (loaded) of the NBS transducer. Adjust the measurement frequency, $f_{m}$, to within $\pm 0.02 \%$ of the specified resonance frequency $f_{s}$, of the transducer using the frequency counter as an indicator. Frequency should be monitored continuously during the test and adjusted, whenever necessary.

5. Set the voltmeter range switch to a suitable low voltage range. Increase the signal generator output to obtain a reading of perhaps 3 to 10 volts. Adjust the controls in the matching-circuit to obtain maximum voltage, being careful not to exceed 3 volts unloaded to 350 volts water loaded (if necessary reduce the signal from the generator).

6. The signal can now be increased to a desired test level. Table A-1 gives the test points (in terms of frequency and voltage) at which measurements are to be made by all participants. The number of measurements to be made at each point is not specified, although a number from 3 to 10 would seem practical.

TABLE A-1

\begin{tabular}{ccc}
\hline $\begin{array}{c}\text { Transducer } \\
\text { No. }\end{array}$ & $\begin{array}{c}\text { Frequency } \\
f_{s}, \mathrm{MHz}\end{array}$ & $\begin{array}{c}\text { Specified } \\
\text { voltages, } V_{s}\end{array}$ \\
\hline $3-16$ & 1.995 & $30-100-300$ \\
$3-18$ & 1.995 & $30-100-300$ \\
$3-20$ & 5.0457 & $12-40-125$ \\
$3-22$ & 5.0148 & $12-40-125$ \\
\hline
\end{tabular}

